\title{
Immune homeostasis to microorganisms in the guts of triatomines (Reduviidae) - A Review
}

\author{
Eloi S Garcia' ${ }^{1,2}{ }^{+}$, Daniele P Castro', Marcela B Figueiredo', Patrícia Azambuja ${ }^{1,2}$ \\ 'Laboratório de Bioquímica e Fisiologia de Insetos, Instituto Oswaldo Cruz-Fiocruz, Av. Brasil 4365, 21045-900 Rio de Janeiro, RJ, Brasil \\ ${ }^{2}$ Instituto Nacional de Ciência e Tecnologia-Entomologia Molecular, Conselho Nacional de Desenvolvimento \\ Científico e Tecnológico, Rio de Janeiro, RJ, Brasil
}

\begin{abstract}
Bacteria, fungi and parasites are in constant contact with the insect gut environment and can influence different aspects of the host gut physiology. Usually, some of these microorganisms develop and survive in the digestive tract. Therefore, the gut environment must be able to tolerate certain populations of these organisms for the establishment of interactions between non-pathogenic bacteria, parasites and the gut. This review provides a brief overview of the biological and molecular mechanisms that microorganisms use to interact with the gut epithelia in mosquitoes and speculates on their significances for the development of bacteria and Trypanosoma cruzi in the guts of triatomines.
\end{abstract}

Key words: gut homeostasis - triatomines - immune reactions - Trypanosoma cruzi - microorganisms

Insects live in complex environments that contain diverse pathogenic organisms, which can reach and infect epithelial surfaces. The most vulnerable surface of an insect is the epithelial surface of the digestive tract, which is in constant contact with an enormous variety of bacteria, fungi and parasites. More than 100 years ago, Metchnikoff (1908) suggested that microflora have beneficial roles in the physiology of gut epithelia and in host fitness. Microorganisms in the gut can establish different interactions with the insects, such as mutual, commensal or pathogenic relationships. An important function of commensal bacteria in the insect gut is to supply essential nutrients to the insect, facilitate digestion and defend the gut surface against opportunistic pathogens, such as parasites and other microorganisms (Dillon \& Dillon 2004, Schaub 2009). In vertebrates and insects, many studies have demonstrated that commensal microbes modulate the expression of several host genes that actively influence the host gut physiology (Koropatnick et al. 2004, Dale \& Moran 2006, Feldhaar \& Gross 2008, Lee 2008, Xi et al. 2008, Dong et al. 2009).

State of the art in Drosophila and vector mosquitoes - Drosophila melanogaster is an excellent model for investigating the complex interactions that occur between microorganisms and the gut interface because the gut epithelium rapidly develops efficient innate immune reactions (Nehme et al. 2007, Ryu et al. 2008, Ha et al. 2009a, b). A signaling network composed of complex positive and negative regulators was described in Drosophila (Leulier \& Royet 2009). These regulators control

Financial support: IOC, CNPq, FAPERJ, PAPES/FIOCRUZ

ESG and DPC contributed equally to this work.

+ Corresponding author: egarcia@ioc.fiocruz.br

Received 19 November 2009

Accepted 12 May 2010 the expression and activation of certain immune reactions that are involved in the production of antimicrobial peptides (AMP), reactive oxygen species (ROS) and reactive nitrogen species (RNS) or intermediates. The activation of certain immune reactions depends on whether the gut has infectious or commensal microorganisms (Hao et al. 2003, Lee 2008, 2009, Leulier \& Royet 2009) (Fig. 1).

AMP production is related to two main distinct pathways: the Toll and immune deficiency (IMD) pathways (Marmaras \& Lampropoulou 2009) (Fig. 2). These pathways are activated by the recognition of pathogenassociated molecular patterns and microbial cell components, which include lipopolysaccharides (LPS) of Gram bacteria, lipoteichoic acid and peptidoglycans (PGN) of $\mathrm{Gram}^{+}$bacteria, $\beta$-1,3-glucans from fungi and glycosylphosphatidylinositol from protozoan parasites (Ashida 1990, Teixeira et al. 2002) (Fig. 3). In D. melanogaster, some studies reported that highly purified LPS did not activate the Toll and IMD pathways (Leulier et al. 2003, Kaneko et al. 2004). The immune activation that occurred in the presence of commercial LPS was attributed to PGN contaminants. Recently, highly purified LPS elicited the expression of immune-related genes in the fat body of Bombyx mori. However, the level of immune activation that was induced by highly purified LPS in $B$. mori was lower than the activation that was caused by crude LPS and PGN (Tanaka et al. 2009).

Toll activation leads to the degradation of the Cactus and Caudal nuclear factor inhibitor kappaBs, which results in the nuclear translocations of dorsal-related immunity factor and Dorsal nuclear transcription factor kappaB (NFkB). Activation of the IMD pathway culminates in the translocation of Relish to the nucleus (Feldhaar \& Gross 2008, Pal \& Wu 2009, Welchman et al. 2009) (Fig. 2). The IMD pathway is conserved in insects and is present in almost all epithelial cells, where it is the only signaling cascade responsible for the production of AMPs (Leulier \& Royet 2009). Aggarwal \& Silverman (2008) observed that the IMD pathway is not activated in the gut epithelia of Drosophila that are maintained under 


\section{HOMEOSTASIS IN THE GUT}

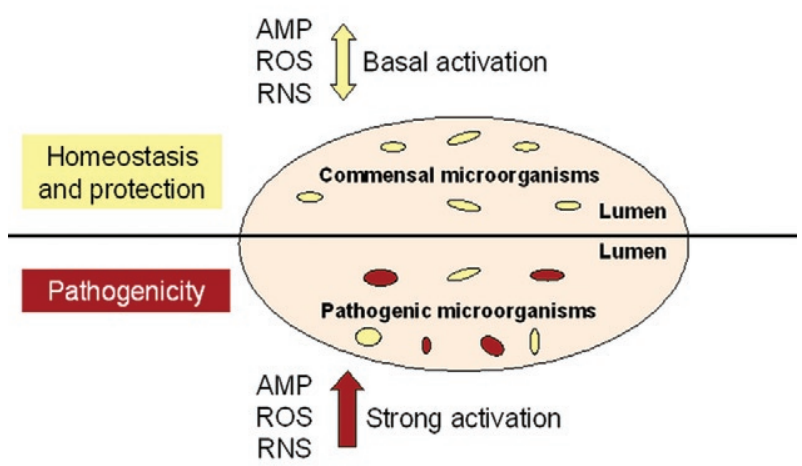

Fig. 1: hypothetic scheme of dipteral immune system: negative and positive balance. Commensal bacteria activate basal production of antimicrobial peptides (AMP), reactive oxygen species (ROS) and reactive nitrogen species (RNS) culminating in gut protection against pathogens and contributing to gut homeostasis. However pathogenic infection (bacteria, parasites, fungi or virus) strongly activates immune responses (AMP, ROS and RNS) reducing pathogenesis in the insect.

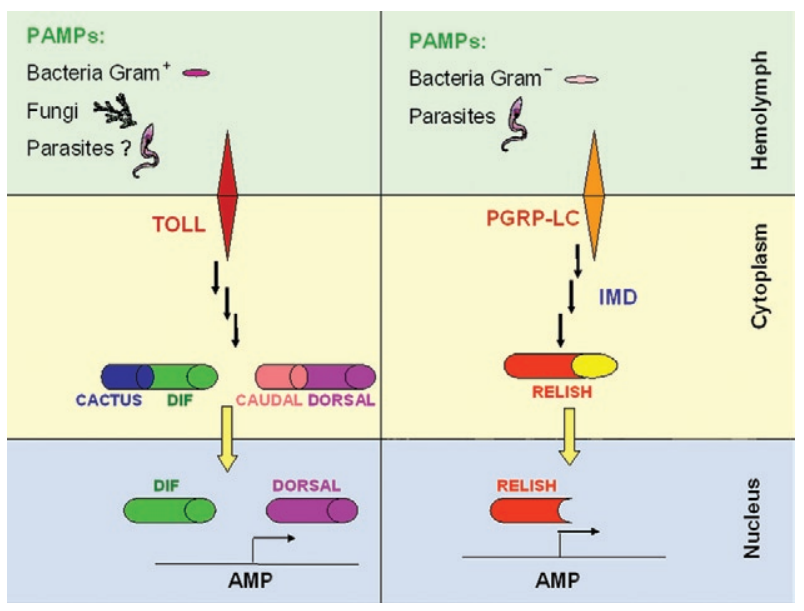

Fig. 2: Toll and immune deficiency (IMD) signaling pathways in diptera. Bacteria $\mathrm{Gram}^{+}$and fungi pathogen-associated molecular patterns (PAMPs) activate Toll receptor. It is not clear yet whether parasites (?) PAMPs activate or not Toll signaling pathway. Gram ${ }^{-}$and parasite PAMPs are recognized by peptidoglycan-receptor protein LC (PGRP-LC) which triggers IMD signaling pathways. The activation leads to nuclear factor inhibitor (Cactus, Caudal and relish) cleavage and translocation of nuclear factors kappaB [dorsal-related immunity factor (DIF), Dorsal and relish] to the nucleus. In the nucleus these factors activate antimicrobial peptide (AMP) transcriptions.

normal conditions, which allows a basal bacterial population to be present. However, under conditions where the Drosophila caudal protein is knocked down, the production of AMPs changes the bacterial populations and causes insect death. In accordance with the observations of Aggarwal and Silverman (2008), Ryu et al. (2008) also observed no differences in the expression of AMPs in the gut, which is mediated by the Caudal homeobox transcription factor, between germ-free animals and animals with gut microflora. This observation indicates that a delicate balance between the effects of the positive and negative NFKBs may be required for the establishment and survival of commensal microorganisms in the gut of Drosophila (Ryu et al. 2008) (Fig. 1).

In the gut of some mosquito vectors, the number of parasites that are ingested with the blood meal decreases before the parasites come into contact with host tissues. The interactions that occur between Anopheles gambiae mosquitoes, Plasmodium parasites and bacterial gut infections are considered elegant models for further investigations (Michel \& Kafatos 2005, Baton et al. 2009, Dong et al. 2009, Meister et al. 2009). Several studies demonstrated that the recognition of PGN, a cell wall component of $\mathrm{Gram}^{+}$and Gram bacteria and bacilli, by the transmembrane PGN recognition protein LC receptor (PGRP-LC) triggers insect immune responses in An. gambiae and D. melanogaster by the IMD pathway. Interestingly, when an An. gambiae blood meal is infected with Plasmodium falciparum, the mosquito defence responses that are typically generated against bacterial infections are also used to destroy the invading parasites (Hao et al. 2001, Dong et al. 2009, Baton et al. 2009, Meister et al. 2009). By performing gene expression analyses, Garver et al. (2009) described genes that were regulated in anopheline species by the activation of Rel2 factors and suggested that the Toll pathway played a more diverse role in mosquito biology than the IMD pathway, which was more specific to immunity. These authors revealed that key mosquito anti-plasmodium factors may be responsible for the IMD-mediated parasite resistance phenotype. Thus, these papers described the regulation of signaling pathways that are activated in response to bacterial and malarial parasite infections and suggested complex interactions exist between parasites, bacteria and the immune systems of insects (Figs 1, 2).

Other factors that are involved in insect gut homeostasis are the antimicrobial ROS and RNS, which produce free radicals that include superoxide $\left(\mathrm{O}_{2}^{-}\right)$and nitric oxide (NO). Nicotinamide adenine dinucleotide phosphate (NAPDH) oxidase and dual oxidase (DUOX) are important for $\mathrm{O}_{2}^{-}$synthesis (Lambeth 2004). $\mathrm{NO}$ is released by inducible isoforms of NO synthase (NOS) and reacts readily with other free radicals, such as $\mathrm{O}_{2}^{-}$, to produce peroxynitrite and other RNS (Fig. 3). Nappi \& Vass (1998) revealed that NO and RNS that are associated with ROS are components of an immune response in insects. Some ROS and RNS have strong antibacterial and antiparasitic properties (Zhu et al. 1992) (Fig. 3). Genetic analyses in Drosophila showed a negative and positive regulation of DUOX-dependent ROS in response to colorizations with commensal and pathogenic microbes. The negative and positive regulations coordinate homeostasis between the insect gut and microbes by efficiently destroying infectious microorganisms while tolerating commensal bacteria (Ha et al. 2009a, b) (Fig. 1).

Ha et al. (2009b) showed that fine regulations of the ROS signaling pathway allow resident bacteria to persist in the gut and prevent the establishment of infectious pathogens. $\mathrm{ROS}$ that are derived from $\mathrm{O}_{2}{ }^{-}$play an essential 


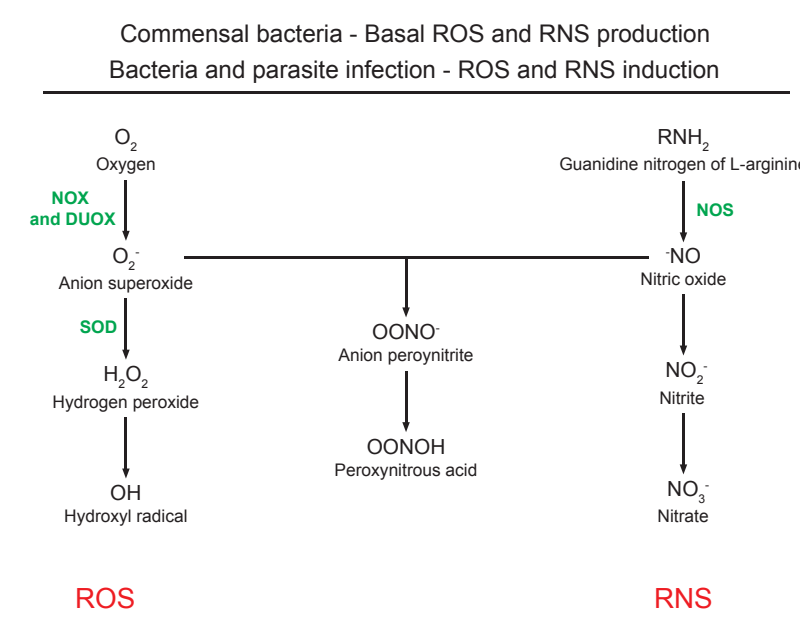

Fig 3: production of main reactive oxygen species (ROS) and reactive nitrogen species (RNS). Commensal bacteria activate basal ROS and RNS production whereas pathogenic bacteria and parasites activate higher production of both immune reactions. Nicotinamide adenine dinucleotide phosphate oxidase (NOX) and dual oxidase (DUOX) cleavage oxygen $\left(\mathrm{O}_{2}\right)$ molecules into anion superoxide $\left(\mathrm{O}_{2}^{-}\right)$which react forming hydrogen peroxide $\left(\mathrm{H}_{2} \mathrm{O}_{2}\right)$ by superoxide dismutase (SOD) action. $\mathrm{H}_{2} \mathrm{O}_{2}$ is hydrolyzed forming hydroxyl radical $(\mathrm{OH})$. RNS are produced through nitric oxide synthase (NOS) activation that cleavages a guanidine nitrogen of L-arginine $\left(\mathrm{RNH}_{2}\right)$ releasing nitric oxide ( $\mathrm{NO})$ that can react forming nitrite $\left(\mathrm{NO}_{2}^{-}\right)$and nitrate $\left(\mathrm{NO}_{3}^{-}\right) \cdot \mathrm{O}_{2}^{-}$and NO can react with each other producing peroxynitrite $\left(\mathrm{OONO}^{-}\right)^{-2}$ and peroxynitrous acid $(\mathrm{OONOH})$.

role in antimicrobial activities and are regulated differently from AMPs in the insect gut. In Drosophila, ROS are constitutively produced in low quantities by the membrane-associated DUOX (Ha et al. 2005) (Fig. 3). Interactions that occur amongst microbiota within the epithelial cells enhance the enzymatic activity of DUOX in the gut (Ha et al. 2009a). These reactions ensure a minimal production of ROS to achieve homeostasis between the gut and microorganisms by efficiently controlling infectious organisms and the proliferation of the microbiota (Fig. 1). DUOX activation is not dependent on PGN (Ha et al. 2009a). However, DUOX transcription is controlled by PGN-dependent and independent pathways. Another important free radical is NO, which is a crucial effector molecule in vertebrate immunology (Fang 1997). The NOS in the gut of Anopheles stephensi is induced by Plasmodium infections and limits the infections (Luckhart \& Rosenberg 1999). Dimopoulos et al. (1998) identified a NOS gene in An. gambiae, which is transcriptionally activated by bacteria and Plasmodium parasites.

Foley and O'Farrell (2003) proposed another role for RNS. These authors suggested that gut bacteria activate NOS, which releases NO into the haemocoel. NO then acts as a signaling molecule to activate immune responses in the haemocytes, which eventually culminates in the production of AMPs by the fat body. Hao et al. (2003) also proposed that reactive intermediates, such as NO and hydrogen peroxide $\left(\mathrm{H}_{2} \mathrm{O}_{2}\right)$, which are synthesized in the proventriculus of the tsetse fly, can easily diffuse into the haemolymph and act as immunological signals that mediate molecular communications between different insect com- partments. Brown et al. (2003) suggested that a messenger system existed between the gut and the haemolymph because the non-invasive gut trypanosome, Crithidia bombi, activated genes that were related to the prophenoloxidase (PPO) system in the haemolymph of the bumble bee.

The PPO cascade, which leads to melanisation, produces highly reactive and toxic compounds (e.g. quinones) and causes the encapsulation of pathogens, which allows insects to clear infections (Garcia et al. 2009). When phenoloxidase (PO) was first described in a midgut homogenate of An. stephensi, the authors observed that the enzyme had characteristics that were different from the PO that was found in the haemolymph (Sidjanski et al. 1997). Recently, POs were shown to be involved in the encapsulation of Plasmodium yoelii in the midgut of Anopheles dirus (Wang et al. 2009). This result confirms that the PO cascade is a humoral immune response that can participate in gut homeostasis.

Models of triatomine immune gut homeostasis - Gut homeostasis is important in triatomines because many bacteria, fungi and parasites, such as Trypanosoma cru$z i$, develop and survive in the digestive tract environment of the triatomine (Garcia et al. 2007, Schaub 2009). The determinations of how the gut tolerates resident microbes and the mechanisms that exist between gut microorganism communities, the gut epithelium, antibacterial defense mechanisms and infections with T. cruzi and Trypanosoma rangeli remain primarily unknown in triatomine vectors.

The majority of triatomine gut bacteria are considered to be commensals (Schaub 2009). However, a large number of bacteria that are present in the gut of the vector after a blood meal have trypanolytic and hemolytic activities (Azambuja et al. 2004). Azambuja et al. (2004) demonstrated that some commensal bacteria are responsible for the establishment of trypanosomes in the digestive tract and for the release of haemoglobin, which is proteolytically digested in Rhodnius prolixus (Fig. 4). Apparently, some bacteria that are present in the gut lumen may attach directly to $T$. cruzi and $T$. rangeli surfaces and kill the parasites (Azambuja et al. 2004, 2005, Castro et al. 2007a, b). Beard et al. (2002) demonstrated that $R$. prolixus that contained transformed symbionts expressed lepidopteron cecropin, which destroys T. cruzi in the gut. Thus, the close association of microorganisms with the gut epithelium suggests that some of the triatomine immune factors that are usually induced by bacterial and/or parasite challenges may be involved in killing bacteria and trypanosomes in the gut and/or the haemolymph.

Lysozymes hydrolyse the 1,4- $\beta$-linkage between $N$ acetylmuramic acid and $N$-acetylglucosamine of bacterial cell wall PGN. Therefore, lysozymes may serve digestive roles, or they may function as immune-related molecules in insects that ingest large numbers of bacteria to prevent the colonisation of the haemocoel by pathogens. In some insects, different isoforms of lysozymes may serve both functions (Ursic-Bedoya \& Lowenberger 2007). Lysozymes are expressed in haemocytes, the fat body and various regions of the digestive tract. Kollien et al. (2003) demonstrated that lysozyme genes are up-regulated in the gut upon Triatoma infestans molting and feeding and are 


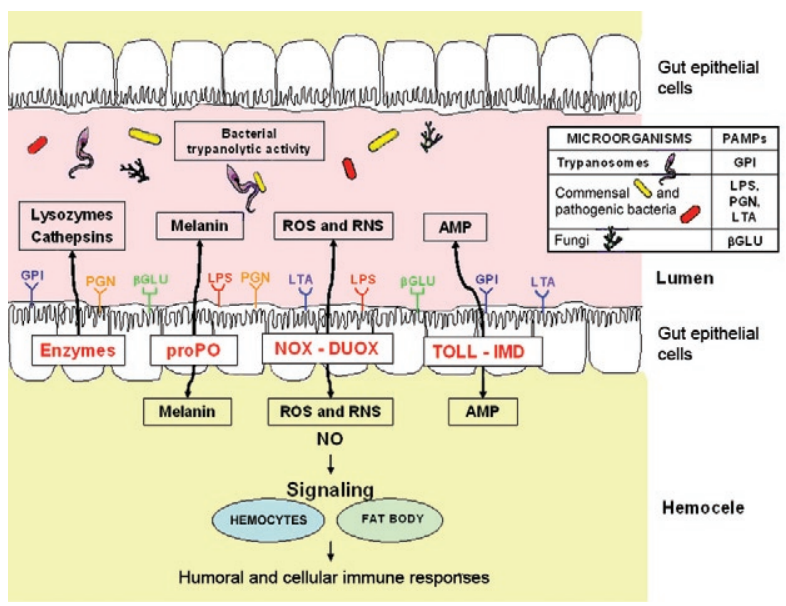

Fig. 4: hypothetic triatomines gut homeostasis. Pathogen-associated molecular patterns (PAMPs) are recognized by gut epithelial receptors leading to activation of signaling pathways culminating in immune responses. Main microorganisms PAMPs are glycosylphospatidylinositol (GPI), lipopolysaccharide (LPS), peptidoglycan (PGN), lipoteichoic acid (LTA) and 1,3 $\beta$-glucan ( $\beta$ GLU). Commensal bacteria lead to low immune response maintaining a basal bacteria population in the gut and protecting the insect from pathogenic microorganisms. Commensal bacteria can also have trypanolytic activity controlling the parasite infection. Pathogenic microorganisms, in contrast to commensal, induce high immune responses. The signaling pathways Toll and immune deficiency (IMD) are related to antimicrobial peptide (AMP) production in the lumen and hemocele. Activation of nicotinamide adenine dinucleotide phosphate (NAPDH) oxidase (NOX) and dual oxidase (DUOX) leads to the production of reactive oxygen species (ROS) and reactive nitrogen species (RNS). Nitric oxide (NO) in the hemocele can function as a signaling molecule inducing immune responses in hemocytes and fat body. Prophenoloxidase (proPO) cascade can also be activated in the epithelial cell and lumen inducing microorganism melanization. Cathepsins and lysozymes also can be activated and act in destroying pathogenic microorganisms.

correlated with an increase in the antibacterial activity in these regions. Araújo et al. (2006) also demonstrated that lysozyme was expressed in the digestive tract of Triatoma brasiliensis, but the expression was higher in the stomach than in the intestine. In R. prolixus stomachs, lysozyme-A genes were predominantly expressed in response to ingestions or haemocelic injections of T. cruzi (Ursic-Bedoya \& Lowenberger 2007). Thus, a direct effect of lysozyme in the establishment of trypanosomal and bacterial infections in the gut may occur (Fig. 4). The presence of some bacteria, which can be controlled by the expression levels of lysozyme, may be deleterious for trypanosomatids (UrsicBedoya et al. 2009).

Other factors that affect trypanosome development are the AMPs of the insect vector and only defensins have been found in triatomines, such as $R$. prolixus and T. brasiliensis (Lopez et al. 2003, Araújo et al. 2006, Waniek et al. 2009). Defensins mainly lyse $\mathrm{Gram}^{+}$bacteria, but they can also kill Gram- bacteria, fungi and parasites (Boulanger et al. 2006). In triatomines, different isoforms of defensins have been observed (Lopez et al. 2003, Araújo et al. 2006, Waniek et al. 2009). In $R$. prolixus, defensin genes are expressed after bacterial inoculations and the activation of immune responses increases the gene transcription in the fat body and the gut (Lopez et al. 2003). The defensin 1 and defensin 4 genes are expressed at high levels in the stomach and very low levels in the intestine, which is similar to the expression of the lysozyme genes (Araújo et al. 2006). Coincidently, the highest bacterial populations are found in the stomach. In contrast to the expression patterns of defensin 1 and defensin 4, the defensin 2 and defensin 3 genes are preferentially expressed in the intestine. Thus, the induction of lysozymes and defensins in the gut suggests that the gut epithelium is a crucial first barrier that helps regulate invading microorganisms (Fig. 4).

Kollien \& Schaub (1998) demonstrated that a higher population density of T. cruzi occurs in the rectum in comparison to the intestine, where proteolysis is restricted and for which the triatomines use cathepsins as digestive proteinases. Experiments using pepstatin, a cathepsin B inhibitor, indicated a small role for proteolytic enzymes in T. cruzi infections (Garcia \& Gilliam 1980). The intestinal cathepsin B mRNA levels did not differ between insects that were infected with T. cruzi and uninfected controls (Ursic-Bedoya $\&$ Lowenberger 2007). However, the activity of cathepsin D was stronger a few days after the ingestion of a blood meal in insects that were infected with T. cruzi when compared to uninfected insects (Borges et al. 2006). Thus, the role of proteolytic enzymes in T. cruzi infections of triatomine guts requires more detailed investigations (Fig. 4).

$\mathrm{PO}$ is a component of the insect immune system that is not present in the intestinal lumen but is present in the cells of the intestinal wall and in haemocytes (summarized by Ratcliffe \& Whitten 2004, Muller et al. 2008). Recent data demonstrate that proPO activity is detected in the lumen and the stomach epithelial cells of R. prolixus (FA Genta, unpublished observations). However, the role of the proPO cascade in gut homeostasis and in the induction of digestive tract humoral immune reactions to parasites and bacteria in triatomines needs to be further investigated (Fig. 4).

Whitten et al. (2007) hypothesized that the expression of NOS genes and the production of NO may be modulated by microorganism infections in different tissues of $R$. prolixus. The most important production of RNS by $R$. proxilus in responses to LPS inoculations occurred in the fat body and haemocytes, whereas intestinal and rectal tissues were the most responsive to T. cruzi and T. rangeli. However, different responses were elicited by these parasites depending on the infection period. During early stage infections, when both species of the trypanosomatids are differentiating epimastigotes that are confined to the stomach, $T$. cruzi upregulated the expression of the NOS gene in the stomach and haemocytes, whereas T. rangeli down-regulated NOS gene expression in these tissues. The induction of extremely high NOS gene expression occurred in the rectum during the late stage of T. rangeli infections. High, sustained concentrations of nitrites/nitrates in the gut wall may have impeded the penetration of $T$. cruzi into the epithelium. In contrast, $T$. rangeli colonized the haemocoel when the midgut nitrite levels decreased (Whitten et al. 2007) (Fig. 4). In the intestine of $R$. prolixus, $T$. rangeli and T. cruzi stimulated large increases in the nitrite levels during the early stages of heavy infections. The nitrite levels in the rectum were significantly high at all stages of the infections with both trypanosome species even before the parasites 
had colonized this tissue (Whitten et al. 2007). The data suggest the NO-mediated immune responses in $R$. prolixus are pathogen-specific and are independently modified by the transcriptional and enzyme levels (Whitten et al. 2007). We hypothesize that NO in triatomines might be a signaling molecule that is activated by gut microorganisms and induces humoral immune responses, such as the production of AMPs, ROS, RNS and cellular immune reactions, such as phagocytosis and haemocyte microaggregation, in the fat body and haemocytes (Fig. 4).

Prostaglandins and leukotrienes, which are formed by the eicosanoid pathway and platelet activation factor (PAF), which is produced by the PAF pathway, are important signaling molecules for cellular immune responses in the haemolymph and the fat body of $R$. prolixus (Figueiredo et al. 2008, Castro et al. 2009, Garcia et al. 2009). Whether these pathways occur in the R. prolixus digestive tract and affect immune homeostasis in the gut is currently under investigation.

Over the past 30 years, $R$. prolixus has been used as a model in our laboratory for investigating several aspects of the interactions that occur between trypanosomes and insect vectors (Garcia et al. 2007, 2009). Like other invertebrate organisms, triatomines contain bacteria and possibly parasites in the gut and develop complex mechanisms to survive with the microorganisms. For the development of parasite control, we can add the importance of resident bacteria with trypanolytic activities to these complex mechanisms. Compounds from bacteria and parasites can induce immune reactions in the insect gut that can have an auxiliary, stock, reserve or backup response that regulates the establishment and the development of the resident bacteria and parasites. The primary immune responses that are induced include lysozymes, AMPs (defensins), proteolytic enzymes (cathepsin D), proPO, ROS and RNS (Fig. 4). The production of these factors might contribute to the balance of microbiota and parasite growth regulation in the guts of triatomines, which ultimately results in the control of pathogenic organisms and leads to resident bacteria and parasites that are tolerant in the epithelia. In these cases, some bacteria escape the immune responses and persist in the gut and the parasites can complete their life cycle in the invertebrate host. Future advances in the genomics and functional genomics of $R$. prolixus will lead to fast developments in this field.

\section{REFERENCES}

Aggarwal K, Silverman N 2008. Positive and negative regulation of the Drosophila immune response. BMB Rep 41: 267-277.

Araújo CA, Waniek PJ, Stock P, Mayer C, Jansen AM, Schaub GA 2006. Sequence characterization and expression patterns of defensin and lysozyme encoding genes from the gut of the reduviid bug Triatoma brasiliensis. Insect Biochem Mol Biol 36: 547-560.

Ashida M 1990. The prophenoloxidase cascade in insect immunity. Res Immunol 141: 908-910.

Azambuja P, Feder D, Garcia ES 2004. Isolation of Serratia marcescens in the midgut of Rhodnius prolixus: impact on the establishment of the parasite Trypanosoma cruzi in the vector. Exp Parasitol 107: 89-96.

Azambuja P, Garcia ES, Ratcliffe NA 2005. Gut microbiota and parasite transmission by insect vectors. Trends Parasitol 21: 568-572.

Baton LA, Robertson A, Warr E, Strand MR, Dimopoulos G 2009. Ge- nome-wide transcriptomic profiling of Anopheles gambiae hemocytes reveals pathogen-specific signatures upon bacterial challenge and Plasmodium berghei infection. BMC Genomics 10: 257.

Beard CB, Cordon-Rosales C, Durvasula RV 2002. Bacterial symbionts of the triatominae and their potential use in control of Chagas disease transmission. Annu Rev Entomol 47: 123-141.

Borges EC, Machado EM, Garcia ES, Azambuja P 2006. Trypanosoma cruzi: effects of infection on cathepsin D activity in the midgut of Rhodnius prolixus. Exp Parasitol 112: 130-133.

Boulanger N, Bulet P, Lowenberger C 2006. Antimicrobial peptides in the interactions between insects and flagellate parasites. Trends Parasitol 22: 262-268.

Brown MJ, Moret Y, Schmid-Hempel P 2003. Activation of host constitutive immune defense by an intestinal trypanosome parasite bumble bees. Parasitology 126: 253-260.

Castro DP, Figueiredo MB, Genta FA, Ribeiro IM, Tomassini TC, Azambuja P, Garcia ES 2009. Physalin B inhibits Rhodnius prolixus hemocyte phagocytosis and microaggregation by the activation of endogenous PAF-acetyl hydrolase activities. $J$ Insect Physiol 55: 532-537.

Castro DP, Moraes CS, Garcia ES, Azambuja P 2007a. Inhibitory effects of d-mannose on trypanosomatid lysis induced by Serratia marcescens. Exp Parasitol 115: 200-204.

Castro DP, Seabra SH, Garcia ES, de Souza W, Azambuja P 2007b. Trypanosoma cruzi: ultrastructural studies of adhesion, lysis and biofilm formation by Serratia marcescens. Exp Parasitol 117: 201-207.

Dale C, Moran NA 2006. Molecular interactions between bacterial symbionts and their hosts. Cell 126: 453-465.

Dillon RJ, Dillon VM 2004. The gut bacteria of insects: nonpathogenic interactions. Annu Rev Entomol 49: 71-92.

Dimopoulos G, Seeley D, Wolf A, Kafatos FC 1998. Malaria infection of the mosquito Anopheles gambiae activates immuneresponsive genes during critical transition stages of the parasite life cycle. EMBO J 17: 6115-6123.

Dong Y, Manfredini F, Dimopoulos G 2009. Implication of the mosquito midgut microbiota in the defense against malaria parasites. PLoS Pathog 5: e1000423.

Fang FC 1997. Perspectives series: host/pathogen interactions. Mechanisms of nitric oxide-related antimicrobial activity. J Clin Invest 99: 2818-2825.

Feldhaar H, Gross R 2008. Immune reactions of insects on bacterial pathogens and mutualists. Microbes Infect 10: 1082-1088.

Figueiredo MB, Genta FA, Garcia ES, Azambuja P 2008. Lipid mediators and vector infection: Trypanosoma rangeli inhibits Rhodnius prolixus hemocyte phagocytosis by modulation of phospholipase A2 and PAF-acetylhydrolase activities. $J$ Insect Physiol 54: 1528-1537.

Foley E, O'Farrell PH 2003. Nitric oxide contributes to induction of innate immune responses to gram-negative bacteria in Drosophila. Genes Dev 17: 115-125.

Garcia ES, Castro DP, Figueiredo MB, Genta FA, Azambuja P 2009. Trypanosoma rangeli: a new perspective for studying the modulation of immune reactions of Rhodnius prolixus. Parasit Vectors 2: 33 .

Garcia ES, Gilliam FC 1980. Trypanosoma cruzi development is independent of protein digestion in the gut of Rhodnius prolixus. J Parasitol 66: 1052-1053.

Garcia ES, Ratcliffe NA, Whitten MM, Gonzalez MS, Azambuja P 2007. Exploring the role of insect host factors in the dynamics of Trypanosoma cruzi-Rhodnius prolixus interactions. J Insect Physiol 53: 11-21.

Garver LS, Dong Y, Dimopoulos G 2009. Caspar controls resistance 
to Plasmodium falciparum in diverse anopheline species. PLoS Pathog 5: e1000335.

Ha EM, Lee KA, Park SH, Kim SH, Nam HJ, Lee HY, Kang D, Lee WJ 2009a. Regulation of DUOX by the Galphaq-phospholipase Cbeta-Ca2 ${ }^{+}$pathway in Drosophila gut immunity. Dev Cell 16: 386-397.

Ha EM, Lee KA, Seo YY, Kim SH, Lim JH, Oh BH, Kim J, Lee WJ 2009b. Coordination of multiple dual oxidase-regulatory pathways in responses to commensal and infectious microbes in Drosophila gut. Nat Immunol 10: 949-957.

Ha EM, Oh CT, Bae YS, Lee WJ 2005. A direct role for dual oxidase in Drosophila gut immunity. Science 310: 847-850.

Hao Z, Kasumba I, Aksoy S 2003. Proventriculus (cardia) plays a crucial role in immunity in tsetse fly (Diptera: Glossinidiae). Insect Biochem Mol Biol 33: 1155-1164.

Hao Z, Kasumba I, Lehane MJ, Gibson WC, Kwon J, Aksoy S 2001. Tsetse immune responses and trypanosome transmission: implications for the development of tsetse-based strategies to reduce trypanosomiasis. Proc Natl Acad Sci USA 98: 12648-12653.

Kaneko T, Goldman WE, Mellroth P, Steiner H, Fukase K, Kusumoto S, Harley W, Fox A, Golenbock D, Silverman N 2004. Monomeric and polymeric Gram-negative peptidoglycan but not purified LPS stimulate the Drosophila IMD pathway. Immunity 20: 637-649.

Kollien AH, Fechner S, Waniek PJ, Schaub GA 2003. Isolation and characterization of a cDNA encoding for a lysozyme from the gut of the reduviid bug Triatoma infestans. Arch Insect Biochem Physiol 53: 134-145.

Kollien AH, Schaub GA 1998. Trypanosoma cruzi in the rectum of the bug Triatoma infestans: effects of blood ingestion by the starved vector. Am J Trop Med Hyg 59: 166-170.

Koropatnick TA, Engle JT, Apicella MA, Stabb EV, Goldman WE, McFall-Ngai MJ 2004. Microbial factor-mediated development in a host-bacterial mutualism. Science 306: 1186-1188.

Lambeth JD 2004. NOX enzymes and the biology of reactive oxygen. Nat Rev Immunol 4: 181-189.

Lee WJ 2008. Bacterial-modulated signaling pathways in gut homeostasis. Sci Signal 27: pe24.

Lee WJ 2009. Bacterial-modulated host immunity and stem cell activation for gut homeostasis. Genes Dev 23: 2260-2265.

Leulier F, Parquet C, Pili-Floury S, Ryu JH, Caroff M, Lee WJ, Mengin-Lecreulx D, Lemaitre B 2003. The Drosophila immune system detects bacteria through specific peptidoglycan recognition. Nat Immunol 4: 478-484.

Leulier F, Royet J 2009. Maintaining immune homeostasis in fly gut. Nat Immunol 10: 936-938.

Lopez L, Morales G, Ursic R, Wolff M, Lowenberger C 2003. Isolation and characterization of a novel insect defensin from Rhodnius prolixus, a vector of Chagas disease. Insect Biochem Mol Biol 33: 439-447.

Luckhart S, Rosenberg R 1999. Gene structure and polymorphism of an invertebrate nitric oxide synthase gene. Gene 232: 25-34.

Marmaras VJ, Lampropoulou M 2009. Regulators and signalling in insect haemocyte immunity. Cell Signal 21: 186-195.

Meister S, Agianian B, Turlure F, Relógio A, Morlais I, Kafatos FC, Christophides GK 2009. Anopheles gambiae PGRPLC-mediated defense against bacteria modulates infections with malaria parasites. PLoS Pathog 5: e1000542.

Metchnikoff E 1908. The prolongation of life: optimistic studies, Putnam, New York, $255 \mathrm{pp}$.
Michel K, Kafatos FC 2005. Mosquito immunity against Plasmodium. Insect Biochem Mol Biol 35: 677-689.

Muller UV, Vogel P, Alber G, Schaub GA 2008. The innate immune system of mammals and insects. In A Egesten, A Schmidt, H Herwald, Contributions to microbiology, Karger, Basel, p. 21-44.

Nappi AJ, Vass E 1998. Hydroxyl radical formation resulting from the interaction of nitric oxide and hydrogen peroxide. Biochim Biophys Acta 1380: 55-63.

Nehme NT, Liégeois S, Kele B, Giammarinaro P, Pradel E, Hoffmann JA, Ewbank JJ, Ferrandon D 2007. A model of bacterial intestinal infections in Drosophila melanogaster. PLoS Pathog 3: e173.

Pal S, Wu LP 2009. Lessons from the fly: pattern recognition in Drosophila melanogaster. Adv Exp Med Biol 653: 162-174.

Ratcliffe NA, Whitten MMA 2004. Vector immunity. In SH Gillespie, GL Smith, A Osbourn, Microbe-vector interactions in vector-borne disease, Cambridge University Press, Cambridge, p. 199-262.

Ryu JH, Kim SH, Lee HY, Bai JY, Nam YD, Bae JW, Lee DG, Shin SC, Ha EM, Lee WJ 2008. Innate immune homeostasis by the homeobox gene caudal and commensal-gut mutualism in Drosophila. Science 319: 777-782.

Schaub GA 2009. Interactions of trypanosomatids and triatomines. Adv In Insect Phys 37: 177-242.

Sidjanski S, Mathews GV, Vanderberg JP 1997. Electrophoretic separation and identification of phenoloxidases in hemolymph and midgut of adult Anopheles stephensi mosquitoes. J Parasitol 83: 686-691.

Tanaka H, Sagisaka A, Fujita K, Kaneko Y, Imanishi S, Yamakawa M 2009. Lipopolysaccharide elicits expression of immune-related genes in the silkworm, Bombyx mori. Insect Mol Biol 18: 71-75.

Teixeira MM, Almeida IC, Gazzinelli RT 2002. Introduction: innate recognition of bacteria and protozoan parasites. Microbes Infect 4: 883-886.

Ursic-Bedoya R, Buchhop J, Lowenberger C 2009. Cloning and characterization of Dorsal homologues in the hemipteran Rhodnius prolixus. Insect Mol Biol 18: 681-689.

Ursic-Bedoya R, Lowenberger CA 2007. Rhodnius prolixus: identification of immune-related genes up-regulated in response to pathogens and parasites using suppressive subtractive hybridization. Dev Comp Immunol 31: 109-120.

Wang Y, Hao H, Qiu ZW, Xu WY, Zhang J, Zhou TL, Zhang XL, Huang FS 2009. Involvement of prophenoloxidases in the suppression of Plasmodium yoelii development by Anopheles dirus. Exp Parasitol 123: 6-10.

Waniek PJ, Castro HC, Sathler PC, Miceli L, Jansen AM, Araújo CA 2009. Two novel defensin-encoding genes of the Chagas disease vector Triatoma brasiliensis (Reduviidae: Triatominae): gene expression and peptide-structure modeling. J Insect Physiol 55: 840-848.

Welchman DP, Aksoy S, Jiggins F, Lemaitre B 2009. Insect immunity: from pattern recognition to symbiont-mediated host defense. Cell Host Microbe 6: 107-114.

Whitten M, Sun F, Tew I, Schaub G, Soukou C, Nappi A, Ratcliffe N 2007. Differential modulation of Rhodnius prolixus nitric oxide activities following challenge with Trypanosoma rangeli, Trypanosoma cruzi and bacterial cell wall components. Insect Biochem Mol Biol 37: 440-452.

Xi Z, Ramirez JL, Dimopoulos G 2008. The Aedes aegypti toll pathway controls dengue virus infection. PLoS Pathog 4: e1000098.

Zhu L, Gunn C, Beckman JS 1992. Bactericidal activity of peroxynitrite. Arch Biochem Biophys 298: 452-457. 